\title{
Novel Clinical and Radiological Findings in a Family with Autosomal Recessive Omodysplasia
}

\author{
Allan Bayat $^{a} \quad$ Morton Dun $^{b} \quad$ Maria Kirchhoff ${ }^{b}$ Finn S. Jørgensen ${ }^{c, d}$ \\ Gen Nishimura ${ }^{e}$ Hanne B. Hove ${ }^{b, f}$ \\ ${ }^{a}$ Department of Genetics and Personalized Medicine, Danish Epilepsy Centre, Dianalund, Denmark; ${ }^{b}$ Department \\ of Clinical Genetics, Copenhagen University Hospital Rigshospitalet, Copenhagen, Denmark; 'Fetal Medicine Unit, \\ Department of Obstetrics and Gynecology, Copenhagen University Hospital Hvidovre, Hvidovre, Denmark;

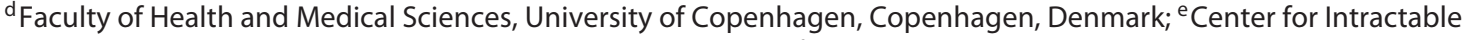 \\ Diseases, Saitama Medical University Hospital, Saitama, Japan; ${ }^{\mathrm{f} D e p a r t m e n t}$ of Clinical Genetics, The RAREDIS \\ Database Section of Rare Diseases, Copenhagen University Hospital Rigshospitalet, Copenhagen, Denmark
}

\section{Established Facts}

- The skeletal hallmarks in autosomal recessive omodysplasia comprise severe shortening and distal tapering of the humeri and femora resulting in a club-like appearance and proximal radioulnar diastasis.

\section{Novel Insights}

- We identified a recurrent and not previously described type of abnormal patterning in all long bones.

\section{Keywords}

GPC6 · Omodysplasia · Radiological feature · Recessive omodysplasia. Skeletal dysplasia

\begin{abstract}
Autosomal recessive omodysplasia (GPC6-related) is a rare short-limb skeletal dysplasia caused by biallelic mutations in the GPC6 gene. Affected individuals manifest with rhizomelic short stature, decreased mobility of elbow and knee joints as well as craniofacial anomalies. Both upper and lower limbs are severely affected. These manifestations contrast with normal height and limb shortening restricted to the arms in autosomal dominant omodysplasia (FZD2-related). Here, we report 2 affected brothers of Pakistani descent from Denmark
\end{abstract}

with GPC6-related omodysplasia, aiming to highlight the clinical and radiological findings. A homozygous deletion of exon 6 in the GPC6 gene was detected. The pathognomonic radiological findings were distally tapered humeri and femora as well as severe proximal radioulnar diastasis. On close observations, we identified a recurrent and not previously described type of abnormal patterning in all long bones.

(c) 2020 S. Karger AG, Basel

Omodysplasia is a rare short-limb skeletal dysplasia with predominant shortening of the upper arms. It was first applied to a series of 5 patients with similar phenotypes by Maroteaux et al. [1989]. The authors also mentioned previous cases described by Barrow and Fitzsim-

\section{KARGER}

(C) 2020 S. Karger AG, Basel

karger@karger.com

www.karger.com/msy
Allan Bayat, MD

Department of Genetics and Personalized Medicine, Danish Epilepsy Centre Kolonivej 1

DK-4293 Dianalund (Denmark)

bayabayabayat@hotmail.com 
mons, [1984] and Viljoen et al. [1987]. Later, omodysplasia was divided into an autosomal recessive (AR) form (GPC6-related, OMIM 258315) and an autosomal dominant (AD) form (FZD2-related, OMIM 164745). Both forms share common craniofacial dysmorphism as well as common skeletal abnormalities of the upper arms. However, AR omodysplasia manifests with severe rhizomelic short stature and both upper and lower limb involvement, while $\mathrm{AD}$ omodysplasia presents with normal height or mild short stature and only the upper limb involvement with distinctively associated shortening of the first metacarpals [Borochowitz et al., 1995; CamposXavier et al., 2009]. Recently, however, some experts prefer to put FZD2-related AD omodysplasia into a group of Robinow syndrome and to apply the term omodysplasia only to GPC6-related AR omodysplasia.

The skeletal hallmarks in AR omodysplasia comprise severe congenital shortening and distal tapering of the humeri and femora, resulting in a club-like appearance and proximal radioulnar diastasis. The elbow and knee joints are restricted. The facial features include frontal bossing, frontal capillary hemangiomas, low-set ears, a flat nasal bridge, anteverted nostrils, and a long prominent philtrum. There have been few reports on associated congenital heart defects, pterygia, craniosynostosis, cryptorchidism, hernias, and cognitive delay [Barrow and Fitzsimmons, 1984; Maroteaux et al., 1989; Borochowitz et al., 1991; Baxova et al., 1994; al Gazali and Abou alAsaad, 1995; Stoll et al., 1995; Masel et al., 1998; Elcioglu et al., 2004; Tan et al., 2005]. To date, approximately 25 cases of AR omodysplasia are known [Maroteaux et al., 1989; al Gazali and Abou al-Asaad, 1995; Borochowitz et al., 1995; Stoll et al., 1995; Masel et al., 1998; Elcioglu et al., 2004; Tan et al., 2005; Albano et al., 2007; CamposXavier et al., 2009]; however, all patients had been described prior to the causal gene discovery [Campos-Xavier et al., 2009]. We report on 2 siblings with AR omodysplasia caused by a homozygous deletion of exon 6 in GPC6. They are the first cases to be published after the clarification of the molecular basis. The aim of this study is to highlight the clinical and radiological findings of these patients.

\section{Case Presentation}

The 2 brothers were born to consanguineous parents (cousins) from Pakistan. The family history was not noteworthy except for the consanguinity. The first child of the couple is a healthy girl. The proband is the second child and was diagnosed clinically with omodysplasia after birth. During the second pregnancy, a routine pre-

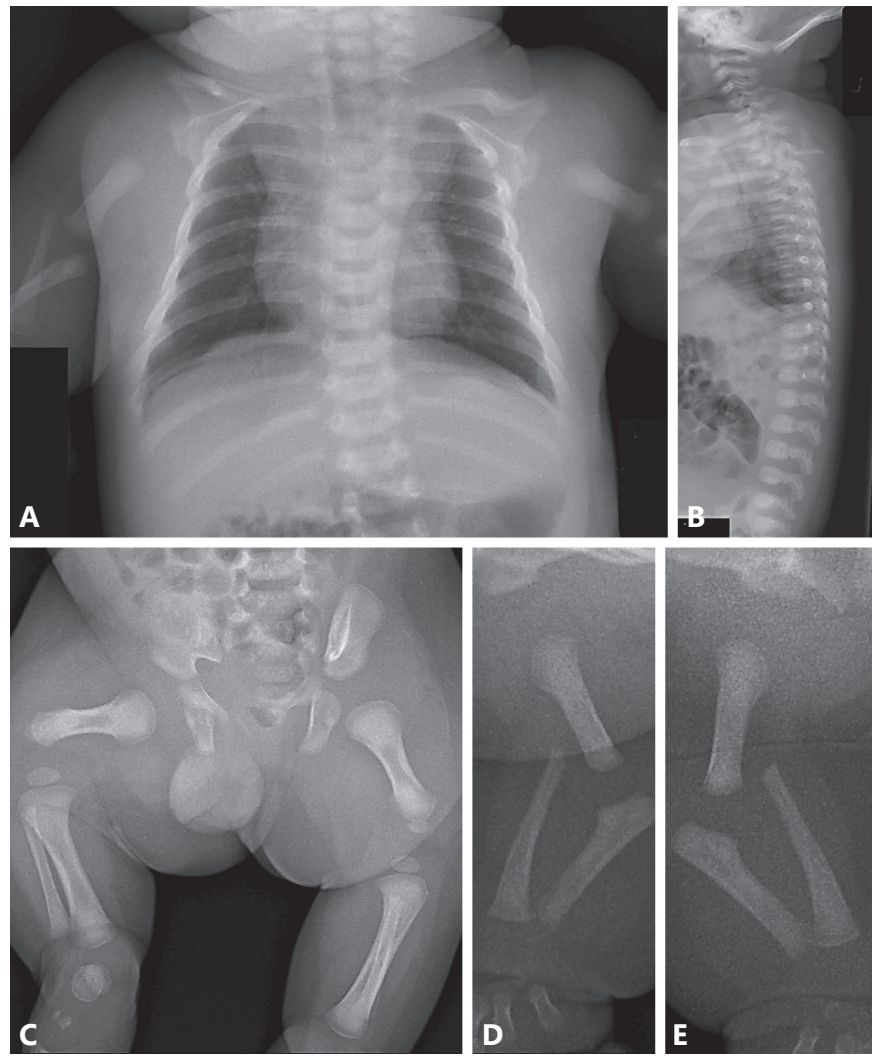

Fig. 1. A-E Skeletal survey of patient 1 after birth showing characteristic features of AR omodysplasia. Features include a broad and short diaphysis and either a distal or a proximal tapering of all long bones. The spine and pelvis were unremarkable (C).

natal ultrasound at 21 weeks' gestation revealed severe rhizomelic shortening of the limbs. The parents were offered a prenatal chromosomal microarray analysis, direct testing for potential mutation in the FGFR3 gene and a gene panel for osteogenesis imperfecta, all of which were performed on a chorionic villus sampling, and no pathogenic abnormalities were identified. The parents did not want an abortion, and at 41 weeks' gestation, the mother gave birth to a boy. Birth weight was 3,300 g (29th centile) and his birth length was $45 \mathrm{~cm}(<0.4$ th centile). His facial features included frontal bossing, a flat nasal bridge, low-set ears, anteverted nostrils, and a long philtrum, but no frontal capillary hemangioma (parental permission to publish pictures was not available). Our patient did not have short first metacarpal bones, which is a feature seen in $\mathrm{AD}$ omodysplasia. He had bilateral cryptorchidism that was eventually corrected surgically at the age of 12 months. He showed no signs of congenital heart defects or craniosynostosis. He learned to sit at the age of 7 months and walk unaided at the age of 15 months. He is currently $3 \frac{1}{2}$ years old and shows no signs of intellectual disability or developmental delay. His height is $74 \mathrm{~cm}(<0.4$ th centile) and the weight is $10 \mathrm{~kg}(<0.4$ th centile). Joint movement of the elbow and knee was restricted.

A skeletal survey was performed shortly after birth and at the age of 3 years. The radiological examination in the neonatal period 

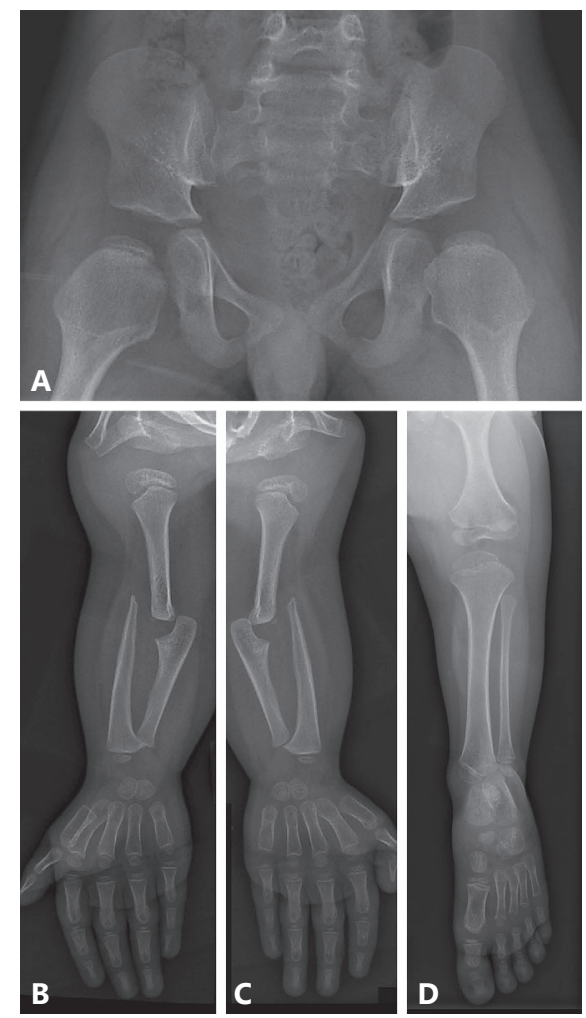

Fig. 2. A-D Skeletal survey of patient 1 at 3 years of age showing the same radiological pattern as that previously seen after birth.

showed short and broad diaphysis in all long bones. The humeri and femora were most severely affected, the ulnae and radii were lesser affected, and the tibiae and fibulae were least affected. The long bones were club shaped varying in appearance due to broadening of one bone end and tapering of the other. The humeri and femora showed distal tapering, the radii and fibulae proximal tapering, while the ulnae and tibiae showed distal tapering. Severe radioulnar diastasis was seen. The proximal tibial epiphyses were larger than normally seen. The acrominal processes of the scapulae were prominent, while the scapular wings were small. The spine, pelvis, hands, and feet were unremarkable (Fig. 1). The follow-up skeletal survey performed at the age of 3 years showed the same radiological pattern as that previously seen (Fig. 2). It was noticeable that the epiphyses of the proximal humeri, proximal femora, and distal femora were flat, while those of the proximal tibiae and distal fibulae were not. The iliac wings were somewhat small along with mild shortening of the greater sciatic notches.

A postnatal chromosomal microarray analysis of a peripheral blood sample of the patient identified an approximately 6-kb homozygous intragenic deletion of exon 6 in GPC6 (NM_005708.5): arr[hg19] 13q31.3(94953093_94958981) $\times 0$. This confirmed the diagnosis of GPC6-related AR omodysplasia. Chromosomal microarray was performed using the Agilent SurePrint G3 Human CGH Microarray kit $2 \times 400 \mathrm{~K}$ (Agilent Technologies, Santa Clara, CA, USA) as previously described [Schejbel et al., 2011]

The second boy (patient 2) with AR dysplasia was born at 39 weeks' gestation. Birth weight was 3,500 g (50th centile), and his
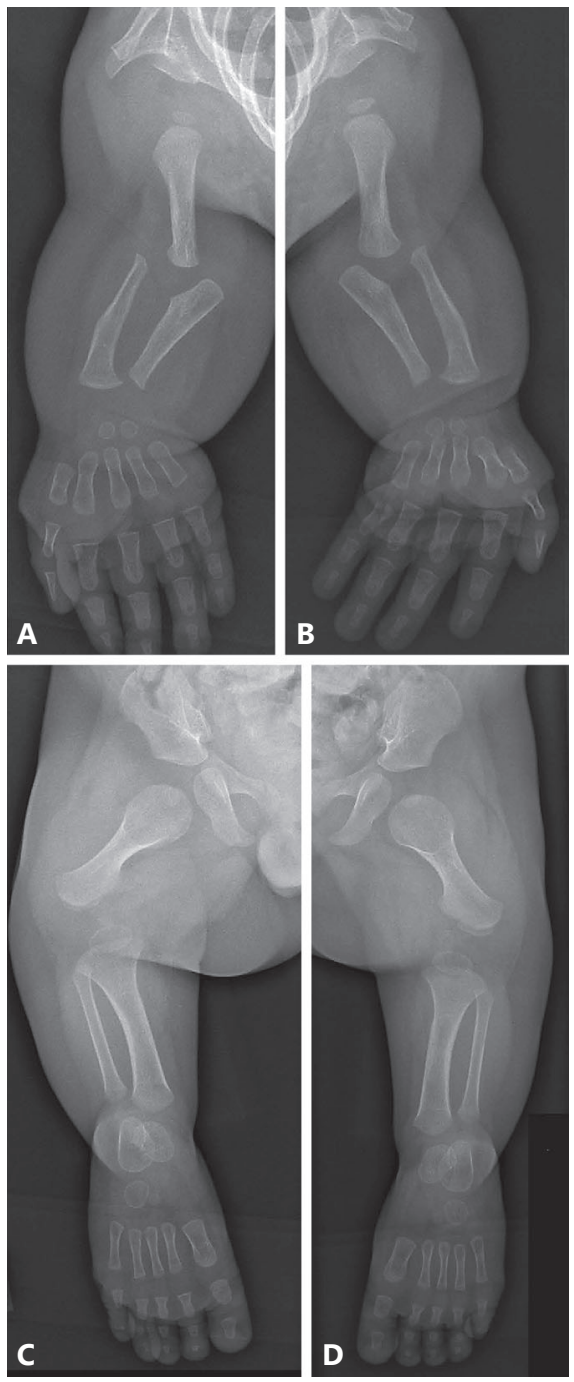

Fig. 3. A-D Skeletal survey of patient 2 at 6 months of age showing the same pattern as that of patient 1 . The proximal tibia showed megaepiphyses at this age (C, D).

birth length was $47 \mathrm{~cm}$ (2nd-9th centile). Prenatal ultrasound had revealed severe short limbs. His facial features also included frontal bossing, a flat nasal bridge, low-set ears, anteverted nostrils, and a long philtrum, but no frontal capillary hemangioma. He had unilateral cryptorchidism that has not yet been operated. Like his brother, he showed no signs of congenital heart defects or craniosynostosis. He is currently 12 months old and has a minor developmental delay in gross motor achievements. His height is $55 \mathrm{~cm}$ $(<0.4$ th centile) and he weighs $5 \mathrm{~kg}(<0.4$ th centile). The elbow and knee joint movement was restricted. A skeletal survey performed at the age of 6 months showed the same pattern as that of patient 1. The proximal tibia showed megaepiphyses at this age (Fig. 3). Postnatal chromosomal microarray analysis of patient 2 showed the same homozygous intragenic deletion in the GPC6 gene as in the older brother (clinical and radiological findings summarized in Table 1). 


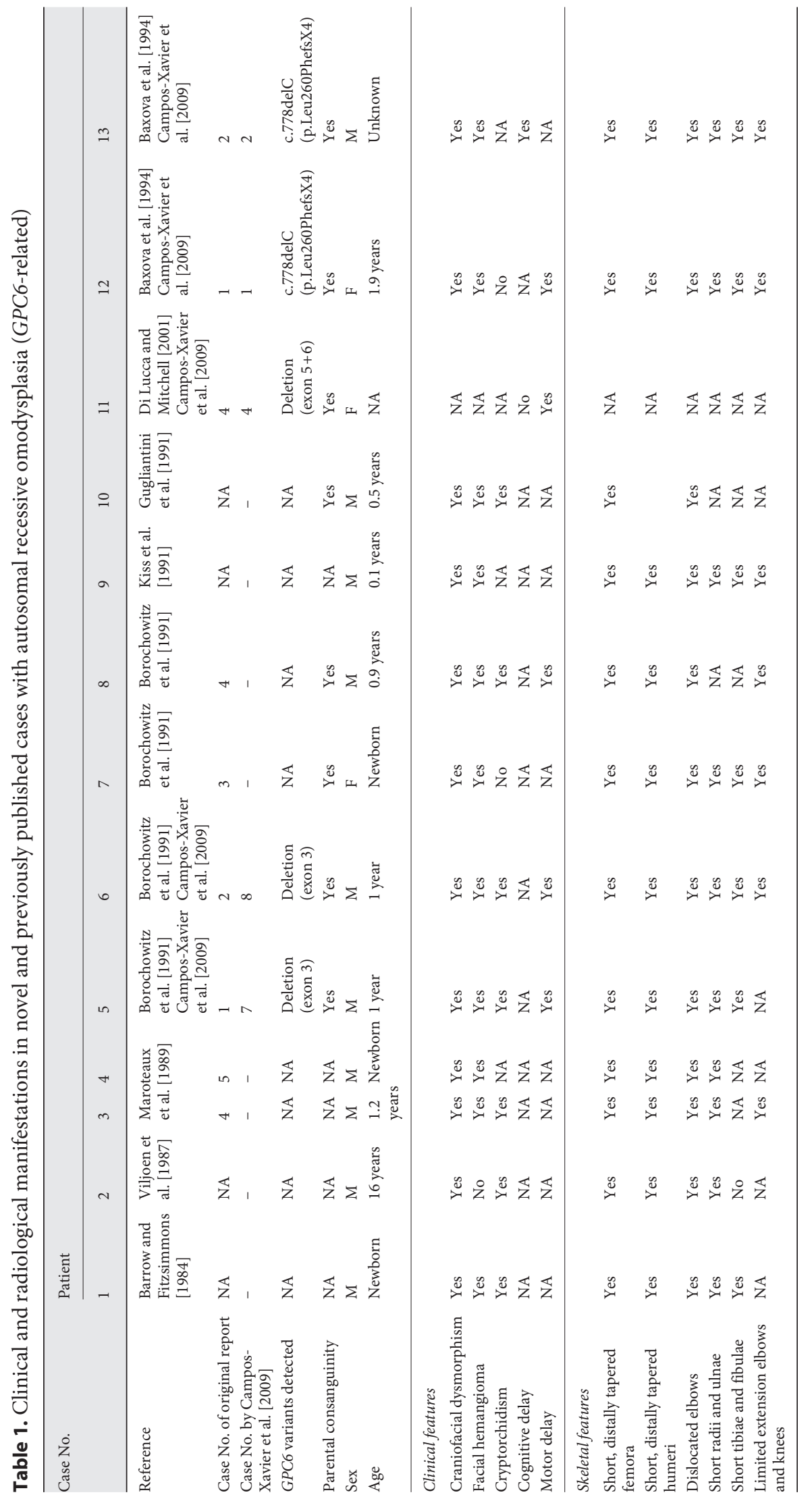




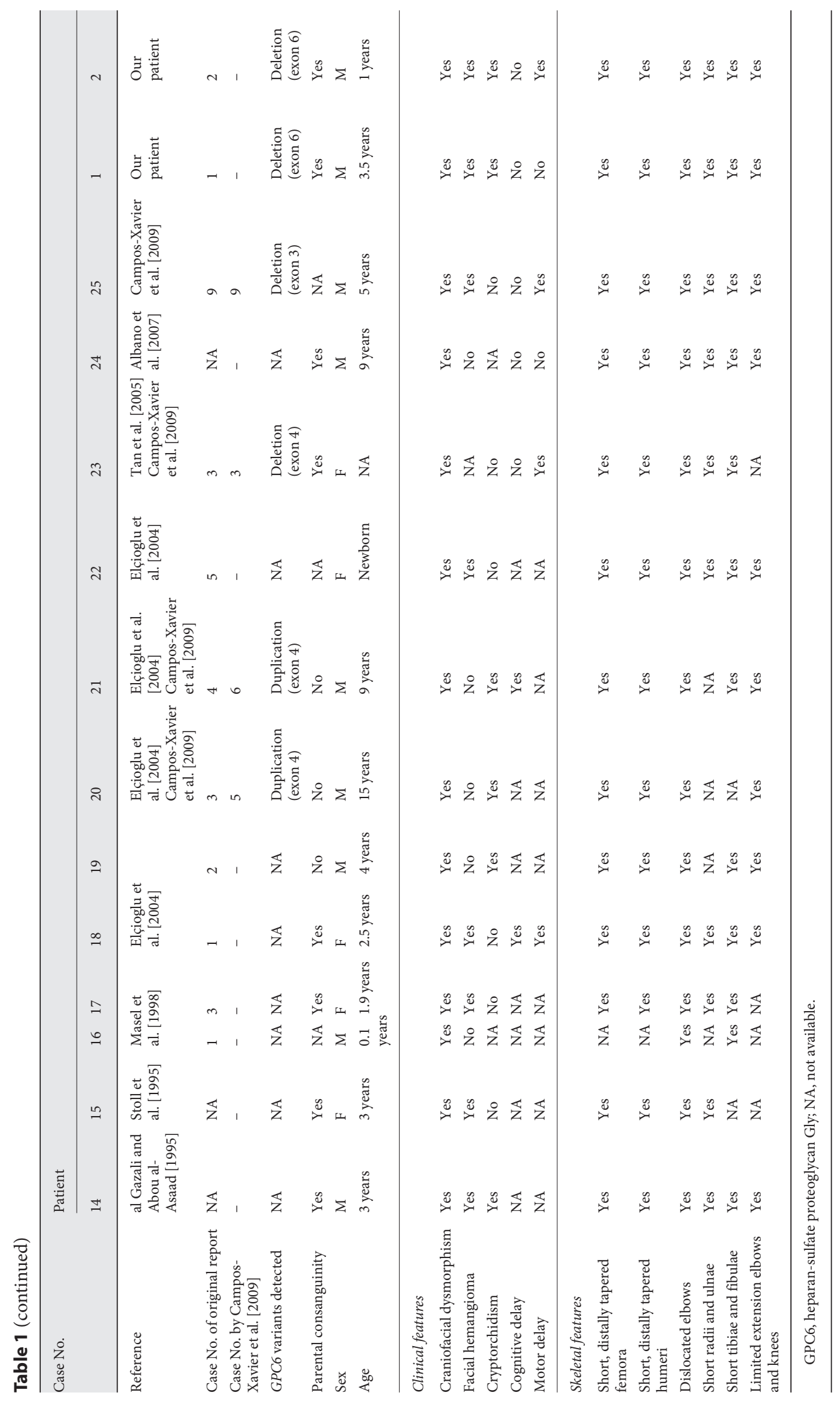




\section{Discussion}

AR omodysplasia has been mapped to chromosome 13 , and loss-of-function mutations in the GPC6 gene (OMIM 604404) encoding glypican 6 (GPC6) were discovered in 2009 [Campos-Xavier et al., 2009]. So far, only genomic rearrangements affecting exons 3, 4, and 6, resulting in premature termination codons and a single base substitution (c.700C $>\mathrm{T}$ ) leading to a nonsense mutation (p.Arg234*) in exon 3, have been shown to cause AR omodysplasia [Campos-Xavier et al., 2009]. A homozygous intragenic deletion of exon 6 as seen in our patients has not been described before. While FZD2 is expressed in the developing face and skeleton and encodes the FRIZZLED2 protein that acts as a Wnt receptor, GPC6 is both expressed in the developing skeleton and neurons [Allen et al., 2012; Saal et al., 2015]. In vitro studies have shown that GPC6 stimulates the activity of various morphogens/growth factors, including Hedgehogs acting on bone growth [Capurro et al., 2017] GPC6 is also expressed by astrocytes in vivo in the developing CNS, particularly in the cerebellum [Allen et al., 2012]. Allen et al. [2012] showed that GPC6 acts as astrocyte-secreted signals sufficient to induce functional synapses between purified retinal ganglion cell neurons. They also showed that depletion of these molecules from astrocyte-conditioned medium significantly reduces its ability to induce postsynaptic activity [Allen et al., 2012]. This potentially explains why a subset of patients with AR omodysplasia have been diagnosed with developmental delay [Nagasaki et al., 2018], while patients with $\mathrm{AD}$ omodysplasia tend to have a normal cognitive development. However, $\mathrm{AD}$ omodysplasia with intellectual disability has been reported by Warren et al. [2018]. In contrary, CamposXavier et al. [2009] argued that the presence of mental retardation could be a potential additional effect of consanguinity in these patients. While patient 1 did not show any signs of developmental delay, his younger brother showed minor developmental delay. These observations may suggest an incomplete penetrance of neurodevelopmental symptoms in GPC6-related AR omodysplasia.

The constellation of distinctive craniofacial dysmorphism, severe short stature, rhizomelic shortening of the limbs and restriction of the elbow and knee joints seen in our patients recapitulated the phenotypic spectrum of AR omodysplasia. The skeletal phenotype of AR omodysplasia was described in detail by Elcioglu et al. [2004]. They reported 5 novel patients, reviewed the literature on the disorder, and emphasized that the skeletal anomalies are confined to the limbs, while the skull, spine, thorax, and the bones of the hand and feet are normal [Elcioglu et al., 2004]. They also mentioned age-dependent evolution of the skeletal changes, i.e., the long bones show much better modeling and less severe shortening with age. The skeletal manifestations of the present cases mostly recapitulated those reported by Elcioglu et al. [2004]; yet, our siblings did not show flat tibial epiphyses, but large proximal tibial epiphyses in infancy, contrasting with their description (for an overview of the radiological findings in patients with AR omodysplasia, see Table 1).

The most striking skeletal change of AR omodysplasia is club-shaped humeri and femora due to hypoplasia of their distal ends and broadening of the proximal ends. Meticulous radiological observations disclosed that other long bones also have a club-like appearance, but to a lesser degree. This distinctive appearance probably represents abnormal pattern formation of the long bones at the early gestational age. Elcioglu et al. [2004] brought attention to the fact that club-like humeri and femora occur in FLNB-related skeletal dysplasias, including Larsen syndrome and atelosteogenesis type 1 and 3. Distal tapering of the humeri and femora can be seen in DTDST-related skeletal dysplasias, including diastrophic dysplasia and atelosteogenesis type 2 . The phenotypic similarities among AR omodyplasia, FLNB-associated dysplasias, and DTDST-associated dysplasias may suggest that glypican 6, filamin B, and sulfated proteoglycans interact with each other at the early patterning of the long bones.

Finally, to the best of our knowledge, only 2 previous cases of prenatally diagnosed AR omodysplasia have been reported in the literature: Borochowitz et al. [1991] reported a prenatal diagnosis of AR omodysplasia made on an ultrasound scan at 17 week's gestation demonstrating short limbs, predominantly humeri and femora, dislocated elbows, and a normal skull and spine, and Tan et al. [2005] reported the earliest clinically diagnosed case of AR omodysplasia at 13 weeks and 3 days. The specific findings at this gestational age were a thickened nuchal translucency $(6.0 \mathrm{~mm})$ with abnormal long bones. Subsequent ultrasounds confirmed these findings, in addition to pronounced edema around the upper trunk [Tan et al., 2005]. In our case, bilateral rhizomelic shortening of the long bones in both pregnancies was helpful to raise a suspicion of skeletal dysplasia. However, due to maternal obesity in both pregnancies, we were unable to make the prenatal detection of proximal radial head dislocation and to delineate the facial profile in detail. With knowledge of the characteristic features of AR omodysplasia outlined in this study, the ultrasonographer may have
88

Mol Syndromol 2020;11:83-89 DOI: $10.1159 / 000506384$
Bayat/Dunø/Kirchhoff/Jørgensen/ Nishimura/Hove 
made a definitive prenatal diagnosis, if the circumstances for the ultrasound examination had been optimal.

\section{Acknowledgment}

The authors thank the family for participating in this study.

\section{Statement of Ethics}

Informed consent for genetic analysis was obtained from the family in compliance with the national ethics regulation.

\section{Diclosure Statement}

The authors have no conflicts of interest to disclose.

\section{References}

Albano LM, Oliveira LA, Bertola DR, Mazzu JF, Kim CA: Omodysplasia: the first reported Brazilian case. Clinics (Sao Paulo) 62:531-534 (2007).

al Gazali LI, Abou al-Asaad F: Autosomal recessive omodysplasia. Clin Dysmorphol 4:52-56 (1995).

- Allen NJ, Bennett ML, Foo LC, Wang GX, Chakraborty C, et al: Astrocyte glypicans 4 and 6 promote formation of excitatory synapses via GluA1 AMPA receptors. Nature 486:410-414 (2012).

Barrow M. Fitzsimmons JS: A new syndrome. Short limbs, abnormal facial appearance, and congenital heart defect. Am J Med Genet 18 : 431-433 (1984).

- Baxova A, Maroteaux P, Barosova J, Netriova I: Parental consanguinity in two sibs with omodysplasia. Am J Med Genet 49:263-265 (1994).

Borochowitz Z, Barak M, Hershkowitz S: Familial congenital micromelic dysplasia with dislocation of radius and distinct face: a new skeletal dysplasia syndrome. Am J Med Genet 39:9196 (1991).

Borochowitz Z, Barak M, Hershkowitz S: Nosology of omodysplasia. Am J Med Genet 58: 377-378 (1995).
Campos-Xavier AB, Martinet D, Bateman J, Belluoccio D, Rowley L, et al: Mutations in the heparan-sulfate proteoglycan glypican 6 (GPC6) impair endochondral ossification and cause recessive omodysplasia. Am J Hum Genet 84:760-770 (2009).

Capurro M, Izumikawa T, Suarez P, Shi W, Cydzik M, et al: Glypican- 6 promotes the growth of developing long bones by stimulating Hedgehog signaling. J Cell Biol 216:29112926 (2017).

Di Luca BJ, Mitchell A: Anaesthesia in a child with autosomal recessive omodysplasia. Anaesth Intensive Care 29:71-73 (2001).

Elcioglu NH, Gustavson KH, Wilkie AO, YukselApak M, Spranger JW: Recessive omodysplasia: five new cases and review of the literature Pediatr Radiol 34:75-82 (2004).

Gugliantini P, Kozlowski K, Cappa M, Orazi C, Borrelli P, Pagnotta G: Rhizomelic bone dysplasia with club-like femora. A distinctive, easily recognisable disease. Case report. Radiol Med 81:550-552 (1991).

Kiss P, Kozlowski K, Zavodi E: Rhizomelic bone dysplasia with club-like femora (case report and confirmation of a syndrome). Australas Radiol 35:266-267 (1991).

Maroteaux P, Sauvegrain J, Chrispin A, Farriaux JP: Omodysplasia. Am J Med Genet 32:371375 (1989).

Masel JP, Kozlowski K, Kiss P: Autosomal recessive omodysplasia: report of three additional cases. Pediatr Radiol 28:608-611 (1998).
Nagasaki K, Nishimura G, Kikuchi T, Nyuzuki H, Sasaki S, et al: Nonsense mutations in FZD2 cause autosomal-dominant omodysplasia: Robinow syndrome-like phenotypes. Am J Med Genet A 176:739-742 (2018).

- Saal HM, Prows CA, Guerreiro I, Donlin M, Knudson L, et al: A mutation in FRIZZLED2 impairs Wnt signaling and causes autosomal dominant omodysplasia. Hum Mol Genet 24: 3399-3409 (2015).

- Schejbel L, Schmidt IM, Kirchhoff M, Andersen $\mathrm{CB}$, Marquart HV, et al: Complement factor $\mathrm{H}$ deficiency and endocapillary glomerulonephritis due to paternal isodisomy and a novel factor $\mathrm{H}$ mutation. Genes Immun 12:90-99 (2011).

Stoll C, Pennerath A, Poirat P: Autosomal recessive omodysplasia. Ann Genet 38:97-101 (1995).

- Tan TY, McGillivray G, Kornman L, Fink AM, Superti-Furga A, et al: Autosomal recessive omodysplasia: early prenatal diagnosis and a possible clue to the gene location. Am J Med Genet A 135:324-327 (2005).

Viljoen D, Goldblatt J, Wallis C, Beighton P: Familial rhizomelic dysplasia: phenotypic variation or heterogeneity? Am J Med Genet 26: 941-947 (1987).

-Warren HE, Louie RJ, Friez MJ, Frías JL, Leroy JG, et al: Two unrelated patients with autosomal dominant omodysplasia and FRIZZLED2 mutations. Clin Case Rep 15:2252-2255 (2018). 